\title{
Hypoxic postconditioning-induced neuroprotection increases neuronal autophagy via activation of the SIRT1/FoxO1 signaling pathway in rats with global cerebral ischemia
}

\author{
JUNJIE LIU ${ }^{1,2}$, ZEHUA GONG ${ }^{1,2}$, JUAN WU ${ }^{3}$, SHAOPENG LIU ${ }^{1}$, XUE WANG $^{3}$, \\ JINGYAO WANG ${ }^{1}$, JIWEI XU ${ }^{2}$, JIANMIN LI ${ }^{1,2}$ and YANING ZHAO ${ }^{3}$ \\ ${ }^{1}$ College of Clinical Medicine, North China University of Science and Technology; ${ }^{2}$ Department of Neurosurgery, \\ The Affiliated Hospital of North China University of Science and Technology; ${ }^{3}$ College of Nursing and \\ Rehabilitation, North China University of Science and Technology, Tangshan, Hebei 063000, P.R. China
}

Received November 9, 2019; Accepted September 2, 2020

DOI: $10.3892 /$ etm.2021.10127

\begin{abstract}
Hypoxic postconditioning (HPC) has been reported to be a beneficial and promising treatment for global cerebral ischemia (GCI). However, its neuroprotective mechanism remains unclear. The aim of the present study was to determine whether the protective effects of HPC in a rat model of GCI were due to the upregulation of autophagy via the silent information regulator transcript-1 (SIRT1)/Forkhead box protein 1 (FoxO1) pathway. Morris water maze test revealed that HPC attenuated cognitive damage in GCI rats. HPC also significantly increased the levels of the autophagy-related protein LC3-II, SIRT1 and FoxO1 compared with those in the GCI group. However, the HPC-induced LC3-II upregulation was blocked by the SIRT1 inhibitor EX527. These results suggested that the beneficial effects of HPC on GCI rats were due to the upregulation of ischemiainduced autophagy and involved the SIRT1/FoxO1 signaling pathway.
\end{abstract}

\section{Introduction}

Cardiac arrest is a leading cause of mortality and disability worldwide (1). Approximately half of the survivors of cardiac arrest experience impairments in memory, attention and executive functioning (2-4). These cognitive deficits are considered

Correspondence to: Dr Jianmin Li, College of Clinical Medicine, North China University of Science and Technology, 57 Jianshe South Road, Tangshan, Hebei 063000, P.R. China

E-mail: linchuangwangyc@ncst.edu.cn

Dr Yaning Zhao, College of Nursing and Rehabilitation, North China University of Science and Technology, 21 Bohai Avenue, Tangshan, Hebei 063000, P.R. China

E-mail: linchuangwangjy@ncst.edu.cn

Key words: hypoxic postconditioning, autophagy, global cerebral ischemia, silent information regulator transcript-1, Forkhead box protein 1 to be primarily due to global cerebral ischemia (GCI) following cardiac arrest, which may induce delayed neuronal cell death in the vulnerable hippocampal CA1 region (5-8). Unfortunately, the treatment options for cardiac arrest-induced neurological impairments are currently limited. A study by Harukuni and Bhardwaj reported that therapeutic hypothermia may have a neurological benefit in cardiac arrest (9), but a recent research failed to confirm any significant benefit on survival or neurological outcome (10). Thus, there is a clear need to identify new potential therapies for reducing brain damage after GCI.

Autophagy plays an important role in neuronal death induced by ischemic brain injury (11-13). Autophagy has been reported to protect neurons from apoptosis, both in a cerebral ischemia model and an oxygen-glucose deprivation mouse model $(14,15)$. Another research demonstrated that autophagy contributed to neuron survival by suppressing apoptosis in a middle cerebral artery occlusion (MCAO) stroke rat model (16). Additionally, Wang et al demonstrated that deletion of $\beta$-arrestin-1, a vital scaffolding protein that interacts with beclin-1 and Vps34 forming a pro-autophagic complex in neurons, hampered autophagosome formation and enhanced neuronal apoptosis (17).

Silent information regulator transcript-1 (SIRT1) is a nicotinamide adenosine dinucleotide $\left(\mathrm{NAD}^{+}\right)$-dependent deacetylase enzyme $(18,19)$. SIRT1 regulates the activity of downstream target genes through deacetylation $(20,21)$. Studies have demonstrated that SIRT1 can attenuate neuronal death and improve cognitive function in mice, indicating that it may also be a novel target for neuroprotection $(22,23)$. EX527 is a potent and selective inhibitor of SIRT1 that exerts different effects in experimental models of diseases and disorders. EX527 has been shown to exert beneficial effects on cerebral ischemia-reperfusion injury and acute lung injury $(24,25)$. The Forkhead box O (FoxO) transcription factor family consists of four members: FoxO1, FoxO3, FoxO4 and FoxO6 (26), among which FoxO1 is widely expressed in brain tissue and may be activated by SIRT1 $(27,28)$. It has been observed that the SIRT1/FoxO1 axis regulates autophagy and promotes phagocytosis of abnormal organelles and proteins (29). When SIRT1 
was silenced, autophagy was inhibited and neuron apoptosis was enhanced (30). These data revealed that the SIRT1/FoxO1 axis is a key regulator of neuron autophagy.

It has been established that hypoxia postconditioning (HPC) may trigger endogenous mechanisms to protect the heart and brain from ischemia-reperfusion injury. Wang et al demonstrated that HPC can significantly reduce the ischemia-reperfusion injury of the heart (31). Similarly, Albrecht et al observed that HPC reduced brain injury caused by perinatal asphyxia (32). Additionally, Nguyen et al reported that HPC reduced brain injury in a MCAO rat model, which indicated a novel approach to the treatment of MCAO-induced brain injury (33). Recent studies have demonstrated that treatment with $8 \%$ oxygen for 120 min applied 1 day after ischemia effectively ameliorated neuronal death induced by GCI in the hippocampal CA1 subregion $(34,35)$. The neuroprotective mechanism induced by HPC after GCI, which may be associated with a variety of intracellular signal transduction pathways, has been investigated, but has yet to be fully elucidated.

The aim of the present study was to examine the efficacy of HPC as a potential novel treatment for brain injury following GCI and to explore the potential mediators through which HPC activates the SIRT1/FoxO1 signaling pathway to promote the survival of neurons in the hippocampal CA1 region after GCI. It was also investigated whether the upregulation of autophagy induced by HPC was caused by the activation of the SIRT1/FoxO1 signaling pathway.

\section{Materials and methods}

Animals and groups. Adult male Sprague-Dawley rats (250-300 g body weight, Animal Center Laboratory of North China University of Science and Technology) were housed in an animal care facility under laboratory conditions of $22-25^{\circ} \mathrm{C}, 50-60 \%$ humidity and a 12 -h light/dark cycle. The rats were given free access to water and food and were allowed to acclimate for 1 week prior to the experimental procedures. All procedures used in the present study were approved by the Institutional Animal Care and Use Committee of North China University of Science and Technology (no. 2015-99), and were conducted in accordance with the guidelines of the National Institutes of Health of the United State of America for animal research. In the present study, as improvement of GCI was an endpoint, determining when the animals should be euthanized was key. Following the study design, the rats were sacrificed under anesthesia once they demonstrated inactivity, difficulty in drinking or eating or abnormal behaviors. Before euthanasia, surgical plane of anesthesia was confirmed by absence of the toe-pinch reflex. Following euthanasia, death was confirmed by checking for absence of heartbeat and respiration.

All rats were randomly divided into five groups ( $n=12$ /group) as follows: i) Sham; ii) GCI; iii) GCI + HPC; iv) $\mathrm{GCI}+\mathrm{HPC}+\mathrm{DMSO}$; and v) $\mathrm{GCI}+\mathrm{HPC}+\mathrm{EX} 527$ groups. EX527 is a potent and selective inhibitor of SIRT1 that exerts different effects in experimental models of diseases and disorders (36). One day prior to the surgery, rats in the GCI + HPC + DMSO and GCI + HPC + EX527 groups underwent lateral ventricle catheterization, after which time $50 \mu \mathrm{l}$ DMSO or EX527 $(1 \mathrm{mmol} / \mathrm{ml})$ were slowly injected into the lateral ventricle $(2 \mu \mathrm{l} / \mathrm{min})$, leaving the needle in for $5 \mathrm{~min}$. With the exception of the sham group, all rats underwent the GCI operation. All rats were weighed daily. One day after the surgery, there were 12 rats in the sham group, 10 in the GCI, 10 in the GCI + HPC, 11 in the $\mathrm{GCI}+\mathrm{HPC}+\mathrm{DMSO}$ and 10 in the GCI + HPC + EX527 group. A total of 7 rats were excluded from the study due to death after the operation. The duration of the experiment was 2 weeks.

GCI model. The four-vessel occlusion rat model of GCI was constructed as previously described (36). The rats were fasted for $10 \mathrm{~h}$ and were not allowed to drink water for $4 \mathrm{~h}$ before the operation. Briefly, after anesthetizing rats with $40 \mathrm{mg} / \mathrm{kg}$ sodium pentobarbital(Diamondback Drugs) by intraperitoneal injection, both vertebral arteries were permanently electrocauterized with an electrocautery needle. Subsequently, the bilateral common carotid arteries (CCAs) were exposed and isolated. After $24 \mathrm{~h}$, the CCAs were exposed and occluded with aneurysm clips for 12 min to induce ischemia. Successful cerebral ischemia was ensured by monitoring the loss of righting reflex, dilated pupils and unresponsiveness to light during GCI. Following the procedure, arterial blood flow was confirmed before the wound was closed with tissue glue. The body temperature of the animals was maintained within the range of $36.5-37.5^{\circ} \mathrm{C}$ during surgery with a baking lamp. For sham-operated animals, all procedures were performed exactly as for ischemic animals, except that the CCAs were not occluded. After modeling, no rats exhibited behavioral defects.

Prior to surgery, healthy animals were observed and assessed twice daily (8:00 a.m. and 8:00 p.m.). During the anesthesia recovery period, the animals were observed continuously until they were able to move, drink and eat independently. After surgery, the animals were observed and assessed every $4 \mathrm{~h}$ to determine whether painkiller administration was required if they were suffering from pain and distress.

HPC protocols. After GCI surgery, the rats were placed into a sealed plastic chamber (BioSpherix, Ltd.), through which air containing $8 \% \mathrm{O}_{2}$ and $92 \% \mathrm{~N}_{2}$ (normal air composition ratio: $21 \% \mathrm{O}_{2}$ and $78 \% \mathrm{~N}_{2}$ ) flowed continuously via a Pro-Ox oxygen controller for $2 \mathrm{~h}$ per day for 3 days. The gas mixture flow was maintained at $200 \mathrm{ml} / \mathrm{min}$ and no more than 4 rats were placed into the chamber at any given time (37). Sham group rats were placed in the chamber with no hypoxia applied.

Morris water maze (MWM) tests. MWM testing was performed in a circular pool (diameter, $180 \mathrm{~cm}$; height, $60 \mathrm{~cm}$; depth, $35 \mathrm{~cm}$ ) filled with opaque water by stirring in milk (temperature, $26 \pm 1^{\circ} \mathrm{C}$ ) to evaluate cognitive function in rats. Briefly, the rats were placed in the water facing the wall at random in one of the four quadrants (I, II, III and IV), located equidistant from one another around the rim of the pool. For each trial, the rat was given a maximum of $90 \mathrm{sec}$ to locate the hidden platform; any rat that failed the mission within $90 \mathrm{sec}$ would be guided to the hidden platform and allowed to stay on the platform for $15 \mathrm{sec}$ before the training 
was terminated. The procedure was repeated from each of the four start locations, within a 4-h interval. The escape latency, representing the average time required to reach the submerged platform, was recorded. The training tests were administered on days 1, 3, 5 and 7 after GCI. Additionally, probe trials were performed $4 \mathrm{~h}$ after the last training session. During the probe trial, the platform was removed from the tank and each rat was allowed to swim freely for $90 \mathrm{sec}$. The number of platform crossings (the number of times passing through the previous location of the platform) was used to evaluate the level of spatial reference memory. Each rat was placed in the pool at the same random start location. All behavioral tracks of the trials were recorded and analyzed using video tracking software (Huaibeizhenghua Biological Equipment Co., Ltd.).

Brain tissue and hematoxylin and eosin $(H \& E)$ staining. Rats were administered an overdose of $4 \%$ sodium pentobarbital $(200 \mathrm{mg} / \mathrm{kg})$ by intraperitoneal injection, and were then transcardially perfused with normal saline $(0.9 \%)$ followed by $4 \%$ paraformaldehyde in $0.1 \mathrm{M}$ sodium phosphate buffer (pH 7.3). The brain was then dissected, postfixed with $4 \%$ paraformaldehyde at $4^{\circ} \mathrm{C}$ for 3 days, embedded in paraffin, cut into $5-\mu \mathrm{m}$ sections and stained with $\mathrm{H} \& \mathrm{E}$. The population of necrotic neurons in the CA1 subfield was counted using a light microscope (BX53; Olympus Corporation) at x400 magnification by two independent investigators who were blinded to the experimental conditions. The mean number of apoptotic neurons was obtained by counting 3 sections per brain and 5 representative fields were randomly selected per section.

Immunohistochemistry staining. Coronal brain sections were prepared as described above. Immunofluorescence staining was performed following a standard protocol as described previously (34). Briefly, the sections were first treated with $3 \%$ hydrogen peroxide for $30 \mathrm{~min}$, followed by $5 \%$ normal serum for $1 \mathrm{~h}$, and they were then incubated overnight at $4^{\circ} \mathrm{C}$ with primary antibodies, including LC3-II (1:300; cat. no. 2275; Cell Signaling Technology, Inc.). Immunopositive cells in which the reaction product was present in the cytoplasmic or nuclear border were quantified under a light microscope at x400 magnification. The number of immunopositive cells was counted in a total of 4 non-repeated random fields $\left(0.037 \mathrm{~mm}^{2} /\right.$ field $\mathrm{x} 4=0.148 \mathrm{~mm}^{2}$ in total $)$ in the CA1 subregions. Data were quantified bilaterally in 4 sections from each rat and assessed in a double-blinded manner.

Sample preparation and western blot analysis. As described previously (38), both hippocampi were rapidly removed from animals under deep anesthesia. The hippocampal CA1 regions were quickly microdissected on an ice pad and immediately frozen in liquid nitrogen. The rat hippocampus was homogenized in RIPA lysis buffer (cat. no. P0013B; Beyotime Institute of Biotechnology) containing PMSF, followed by centrifugation at $12,000 \mathrm{x}$ g for $10 \mathrm{~min}$ at $4^{\circ} \mathrm{C}$. Protein concentrations were determined using a bicinchoninic acid protein assay kit (Beyotime Institute of Biotechnology). A total of $30 \mu \mathrm{g}$ of protein per lane was separated by $12 \%$ SDS-PAGE and transferred onto PVDF membranes. The membranes were blocked

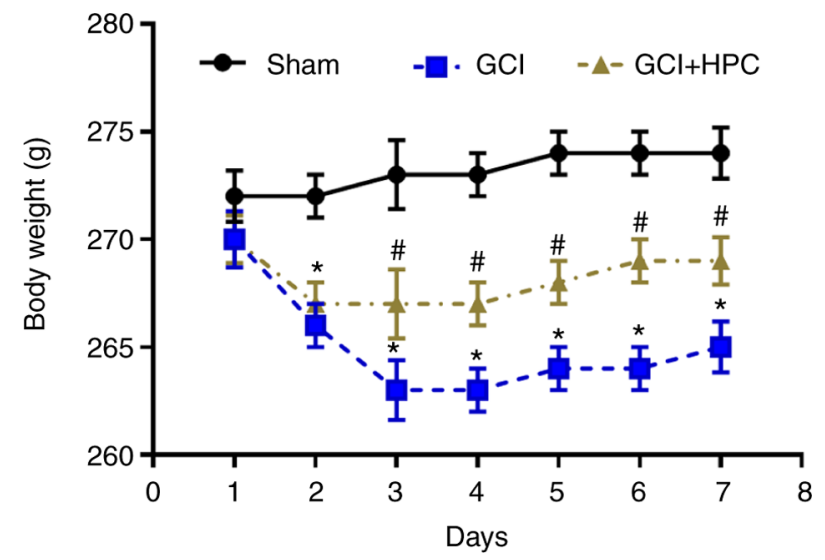

Figure 1. Effects of HPC on GCI-induced weight loss. Data are presented as the mean \pm SD. ${ }^{*} \mathrm{P}<0.05$ vs. sham group; ${ }^{\#} \mathrm{P}<0.05$ vs. GCI group. GCI, global cerebral ischemia; HPC, hypoxic postconditioning.

with 3\% BSA (Sigma-Aldrich; Merck KGaA) in Tris-buffered saline at room temperature for $30 \mathrm{~min}$ and incubated overnight at $4^{\circ} \mathrm{C}$ with primary antibodies against mammalian target (LC3-II; 1:200; cat. no. 3868; Cell Signaling Technology, Inc.), SIRT1 (1:500; cat. no. 9475; Cell Signaling Technology, Inc.), FoxO1 (1:500; cat. no. 2880; Cell Signaling Technology, Inc.) or actin (1:1,000, cat. no. 4967; Cell Signaling Technology, Inc.), followed by incubation with horseradish-peroxidase conjugated anti-rabbit IgG (1:2,000; cat. no. 5127; Cell Signaling Technology, Inc.) for $1 \mathrm{~h}$ at room temperature. Protein signals were detected using an enhanced chemiluminescence system (EMD Millipore) and quantified using Quantity-One software, version 4.6.3 (Bio-Rad Laboratories, Inc.). Actin was used as a protein-loading control.

Statistical analysis. IBM SPSS 22.0 (IBM Corp.) and GraphPad Prism 8.0 (GraphPad Software, Inc.) were used to analyze the data. All data are expressed as mean \pm standard deviation and differences between three or more groups were analyzed by one-way ANOVA. Repeated measures ANOVA was used to compare the weight loss and cognitive results. Tukey's post hoc test was applied where there was a significant difference. $\mathrm{P}<0.05$ was considered to indicate statistically significant differences.

\section{Results}

HPC ameliorates GCI induced weight loss in rats. Rats in the GCI group exhibited more notable weight loss compared with those in the sham group $(\mathrm{P}<0.05)$; however, HPC attenuated this tendency in rats with GCI at 2 days after surgery $(\mathrm{P}<0.05$; Fig. 1).

HPC ameliorates GCI induced cognitive function decline in rats. Cognitive function was determined using the MWM test and statistical analysis was performed using repeated measures ANOVA. During training, significant differences in performance were observed. Rats in the GCI group exhibited a longer escape latency compared with those in the sham group $(\mathrm{P}<0.05)$, and the escape latency was shortened by treatment with HPC $(\mathrm{P}<0.05$; Fig. 2A). Repeated measures 

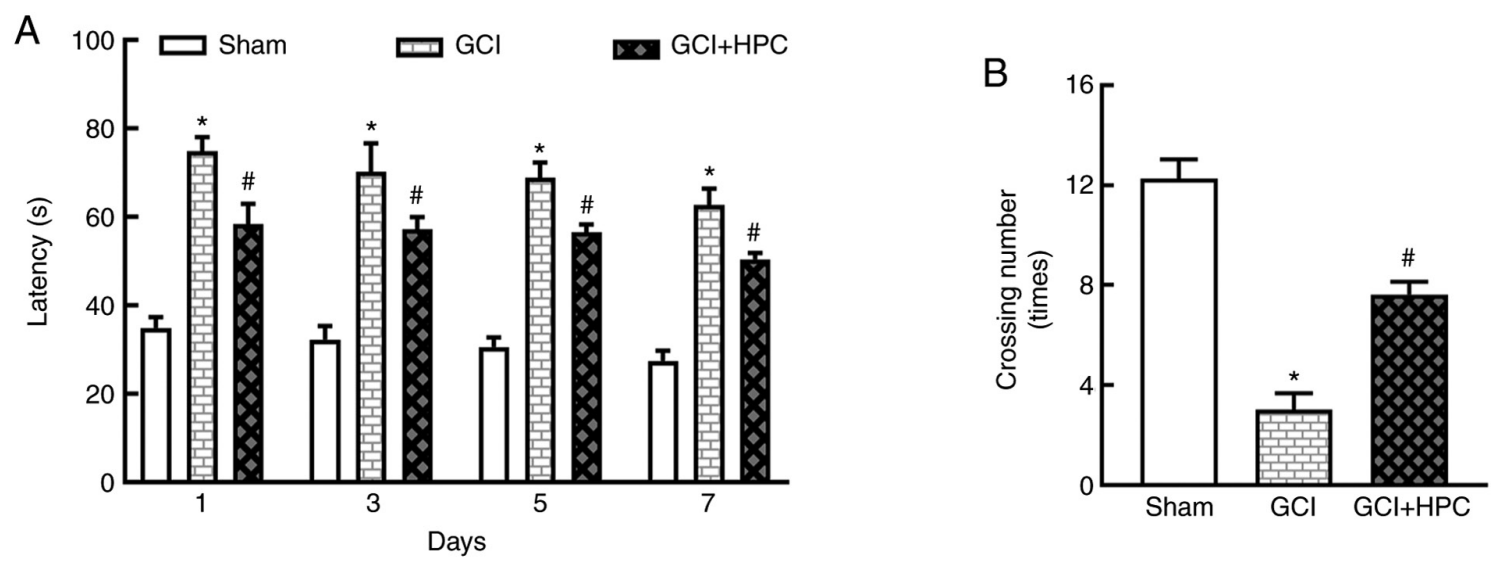

Figure 2. Effects of HPC on GCI cognitive decline in the MWM tests. (A) Escape latency (time taken to locate the submerged platform) in the MWM test. (B) Number of platform crossings in the MWM test. Data are presented as the mean $\pm \mathrm{SD}$. ${ }^{\prime} \mathrm{P}<0.05$ vs. sham group; ${ }^{*} \mathrm{P}<0.05$ vs. GCI group. GCI, global cerebral ischemia; HPC, hypoxic postconditioning; MWM, Morris water maze.

A

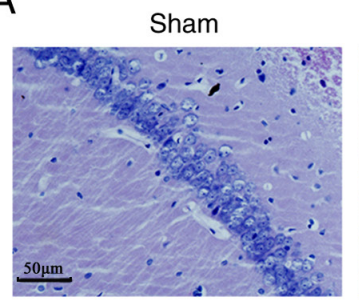

$\mathrm{GCl}$

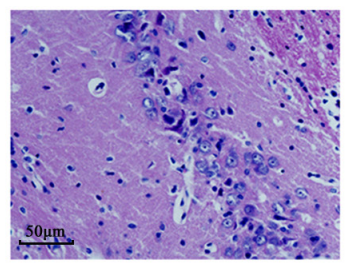

$\mathrm{GCl}+\mathrm{HPC}$

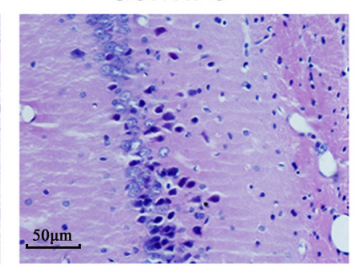

B

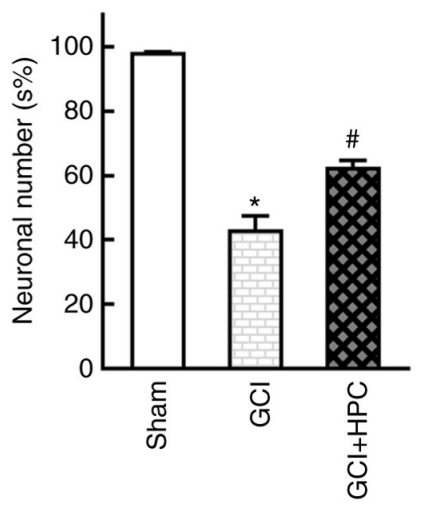

Figure 3. Effects of HPC on neuronal survival in the CA1 region of the hippocampus. (A) Hippocampal neuron survival was detected using hematoxylin and eosin staining (magnification, $\mathrm{x} 400$; scale bars, $50 \mu \mathrm{m}$ ) and (B) the relative number of surviving neurons was obtained. Data are presented as the mean \pm SEM. ${ }^{*} \mathrm{P}<0.05$ vs. sham group; ${ }^{\mathrm{P}}<0.05$ vs. GCI group. GCI, global cerebral ischemia; HPC, hypoxic postconditioning.

ANOVA revealed that the total effect was not statistically significant $(\mathrm{F}=2.874, \mathrm{P}=0.1666)$; however, the groups effect was significant $(\mathrm{F}=918.3, \mathrm{P}=0.000)$. In the probe test, the number of platform crossings was significantly lower in the GCI group compared with that in the sham group $(\mathrm{P}<0.05)$. HPC increased the number of platform crossings compared with the GCI group $(\mathrm{P}<0.05$; Fig. $2 \mathrm{~B})$. These results indicated that HPC ameliorated the impairment of cognitive function induced by ischemia.

HPC promotes neuronal survival in rats with GCI. Histopathological abnormalities were observed in the hippocampus of the rats, and GCI group rats exhibited marked neuronal loss and degeneration of neuronal structure in the CA1 region. HPC attenuated this neuronal loss and reversed the structural injury, as reflected by the number and morphology of neurons on HE staining examination $(\mathrm{P}<0.05)$. These results suggested that HPC inhibited hippocampal neuron death in rats with GCI (Fig. 3).

HPC increases autophagy in the hippocampal CAl area in rats with GCI. To identify autophagy, the expression of the autophagy marker protein, LC3-II, was detected in the hippocampus using immunohistochemistry and western blotting at
1, 3 and 7 days after GCI surgery. Fewer positive cells were visible in the hippocampal CA1 region of the rats in the sham group under an optical microscope (Fig. 4). By contrast, there were abundant positive cells in the GCI group. Furthermore, the number of LC3-II positive cells was significantly increased in the GCI + HPC group compared with that in the GCI group $(\mathrm{P}<0.05)$, which indicated that autophagy was activated further. Low levels of LC3-II were present in the hippocampal CA1 region of the rats in the sham group. However, the expression levels of LC3-II were significantly increased in the GCI group $(\mathrm{P}<0.05)$ and increased further in the GCI + HPC group $(\mathrm{P}<0.05)$. These results suggested that $\mathrm{HPC}$ upregulated autophagy in rats with GCI (Fig. 4).

HPC upregulates autophagy via a SIRT1/FoxO1-dependent pathway in rats with GCI. The role of the SIRT1/FoxO1 axis in GCI-induced autophagy was assessed by measuring the relative protein levels of SIRT1 and FoxO1 in the four groups. EX527, a SIRT1 inhibitor, was able to reduce the expression levels of SIRT1 and FoxO1 $(\mathrm{P}<0.05)$; it simultaneously decreased the expression levels of LC3-II in GCI rats compared with those in GCI + HPC + DMSO group rats $(\mathrm{P}<0.05$; Fig. 5). These results indicated that HPC may upregulate autophagy through a SIRT1/FoxO1-dependent pathway in rats with GCI. 
A

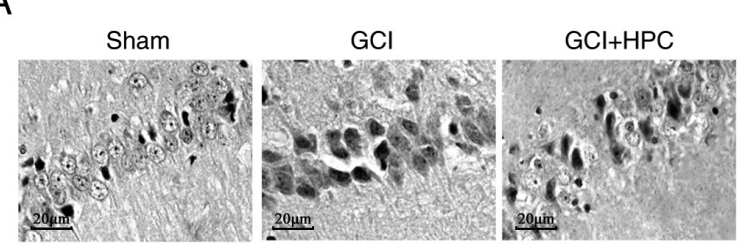

C

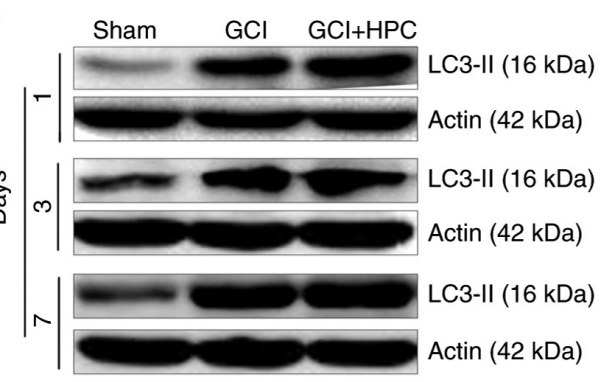

B

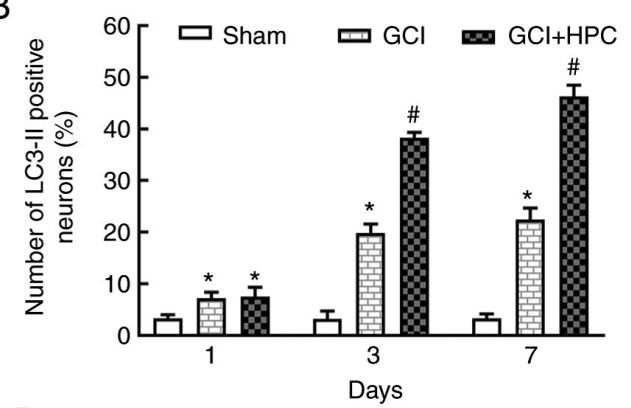

D

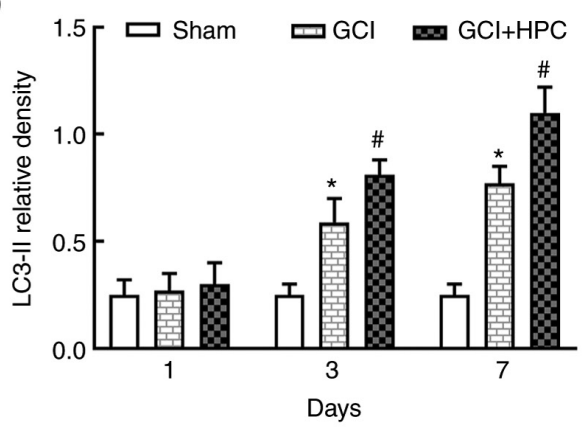

Figure 4. Effects of HPC on autophagy in the CA1 area of the hippocampus. (A) Immunohistochemistry staining (magnification, $\mathrm{x} 400$; scale bars, $20 \mu \mathrm{m}$ ) of LC3-II in the CA1 area. The positively stained cells were observed in the sham, GCI and GCI + HPC groups. (B) Quantitative analysis of the number of positive cells per field at 1,3 and 7 days after GCI surgery. (C) The protein expression levels of LC3-II in the CA1 area were examined by western blot analysis (D) Relative densitometry analysis of LC3-II protein bands. Data are presented as mean $\pm \mathrm{SD}$. ${ }^{*} \mathrm{P}<0.05$ vs. sham group; " $\mathrm{P}<0.05$ vs. GCI group. GCI, global cerebral ischemia; HPC, hypoxic postconditioning; LC3-II, light chain 3-II.
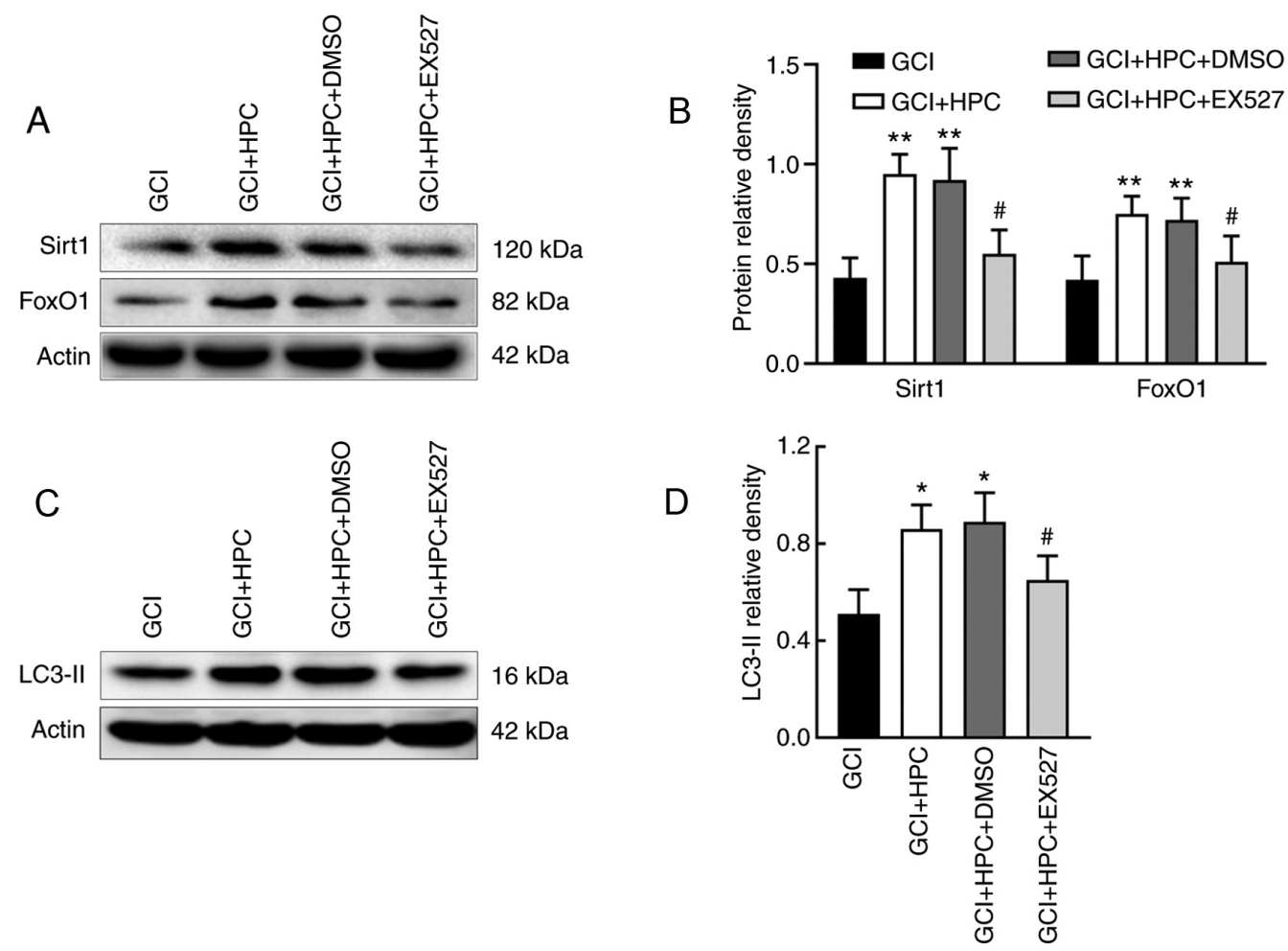

Figure 5. HPC and EX527 affect the expression of SIRT1, FoxO1 and LC3-II. (A) The protein expression levels of SIRT1 and FoxO1 in CA1 were measured by western blot analysis. (B) Relative densitometry analysis of SIRT1 and FoxO1 protein bands. (C) The protein expression levels of LC3-II in CA1 were measured by western blot analysis. (D) Relative densitometry analysis of LC3-II protein bands. Data are presented as mean \pm SD. ${ }^{*} \mathrm{P}<0.05$ vs. GCI group, ${ }^{* * *} \mathrm{P}<0.01$ vs. GCI group; ${ }^{\text {P }}<0.01$ vs. GCI + HPC group. GCI, global cerebral ischemia; HPC, hypoxic postconditioning; LC3-II, light chain 3-II; SIRT1, silent information regulator transcript-1; FoxO1, Forkhead box protein 1; EX527, SIRT1 inhibitor. 
A

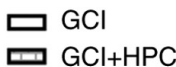

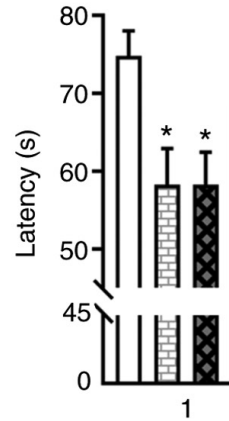

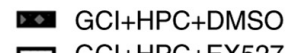

G $\mathrm{GCl}+\mathrm{HPC}+\mathrm{EX} 527$

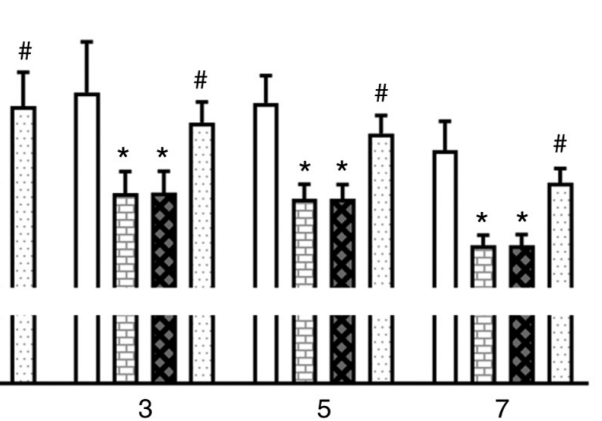

B

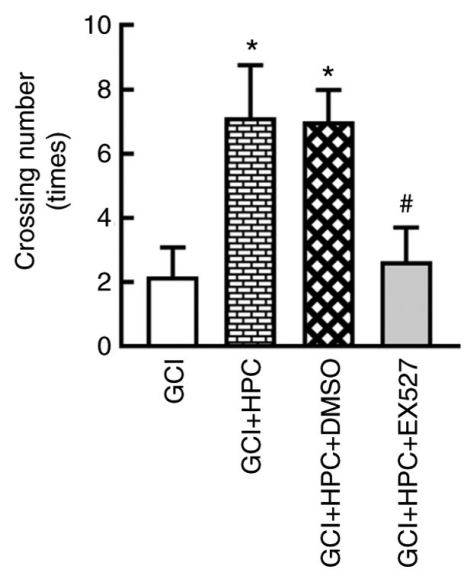

Figure 6. HPC and EX527 affect cognitive function impairment in rats with GCI. (A) Escape latency in the MWM test. (B) Number of platform crossings in the MWM test. Data are presented as the mean \pm SD. ${ }^{~} \mathrm{P}<0.05$ vs. GCI group; ${ }^{~} \mathrm{P}<0.05$ vs. GCI + HPC group. GCI, global cerebral ischemia; HPC, hypoxic postconditioning; MWM, Morris water maze; EX527, SIRT1 inhibitor.

cognitive function was determined using the MWM test. As shown in Fig. 6, EX527 reversed the decrease in escape latency and the increase in the number of platform crossings induced by HPC. Repeated measures ANOVA revealed that the total effect was not statistically significant $(\mathrm{F}=3.483, \mathrm{P}=0.1438)$; however, the groups effect was significant $(\mathrm{F}=325.3, \mathrm{P}=0.001)$. These results indicated that HPC ameliorated cognitive function via a SIRT1/FoxO1-dependent pathway in rats with GCI.

\section{Discussion}

Similar to humans, experimental GCI induced in animal models has been shown to result in selective and delayed neuronal death of pyramidal neurons in the hippocampal CA1 region, as well as cognitive impairment; therefore, experimental GCI in animals has become a valuable model for the study of GCI $(39,40)$. The present study demonstrated that the cognitive function of rats was impaired and weight loss was obvious after GCI. GCI group rats exhibited marked neuronal loss and degeneration of neuronal structure in the CA1 region, whereas HPC was able to alleviate these changes. These results are similar to those of earlier studies reporting that HPC treatment may confer neuroprotection against brain ischemia or injury (35,41-43). In addition, by measuring the expression of the autophagy marker protein LC3-II in the hippocampus, it was observed that there were more positive cells in the hippocampal CA1 region of the GCI group compared with that of the sham group. Interestingly, HPC can upregulate the expression of LC3-II. Further studies have found that HPC can upregulate autophagy by activating the SIRT1/FoxO1 signaling pathway, thereby reducing neuronal death and ameliorating cognitive function impairment. Taken together, all the aforementioned findings indicate that HPC treatment may represent a new therapeutic modality for multiple neurological disorders.

Autophagy is a highly regulated process that involves the degradation of cytoplasmic macromolecules and organelles. In mammalian cells, this catabolic mechanism utilizes the lysosomal system and has a homeostatic function in normal cell growth and development, helping to maintain a balance between the synthesis, degradation and subsequent recycling of cellular products (44). Autophagy is a programmed and physiologically conserved self-degradation process (45) and disruption of autophagy may have detrimental consequences. It was previously demonstrated that autophagy prevents neuronal death in response to GCI (46). In the present study, a specific marker for autophagosomes, LC3-II, was used to test whether autophagic activity can be induced by HPC treatment. As shown in Fig. 4, the expression of LC3-II was markedly elevated following GCI, and this phenomenon was enhanced by HPC treatment, which suggests that HPC treatment effectively suppresses GCI-induced neuronal death in the hippocampal CA1 region by further activating autophagy. Recently, it was also demonstrated that pre-treatment with inhibitors of autophagy markedly increased the infarct area in neonatal and adult rats with MCAO (38). Therefore, it may be hypothesized that GCI activates neuronal autophagy, but this is not sufficient to cause the self-digestion of damaged organelles and to induce neuronal death, which is particularly crucial during neurodegeneration. Furthermore, the neuroprotective effect of HPC may be associated with further upregulation of GCI-induced neuronal autophagy in the hippocampal CA1 region.

As previous studies have demonstrated that various signaling pathways contribute to neuronal autophagy, it was inferred that neuronal autophagy may be the downstream target of SIRT1/FoxO1 in GCI rats $(47,48)$. SIRT1 overexpression may promote autophagy of neurons by regulating FoxO1-mediated target genes, which eventually results in cell autophagy, indicating that the SIRT1/FoxO1 axis may be one of the pathways involved in neuroprotection (49). In the present study, western blot analysis demonstrated that SIRT1 expression was upregulated by HPC treatment, leading to an increasing expression level of FoxO1. The increased expression of SIRT1, FoxO1 and LC3-II was markedly rescued by treatment with a selective SIRT1 inhibitor. Surprisingly, the ability of HPC to upregulate autophagy was suppressed, and its ability to improve cognitive function was also inhibited. These results suggest that SIRT1/FoxO1 may be a key pathway involved in HPC-induced neuron autophagy. This is in accordance with the findings of Jiang et al (50), who also demonstrated that FoxO1 can upregulate autophagy to reduce neuron death in vascular dementia rats. 
In addition, Wu et al (51) also reported that SIRT1 upregulated the deacetylation of FoxO1 and promoted its nuclear translocation, thus increasing cell autophagy to resist ischemia-induced damage. These findings suggest that deacetylation of FoxO1 by activated SIRT1 is one of the mechanisms through which HPC exerts its effects. Although it has been demonstrated that SIRT1 activation is a promising candidate for treating or preventing several diseases and disorders, some studies suggest that SIRT1 inhibition may have beneficial effects on some pathological conditions. For example, Nikseresht et al (24) demonstrated that the mortality and cerebral infarct volume of rats can be reduced in a focal model of cerebral ischemia-reperfusion by using EX527 to inhibit SIRT1. In addition, Jiang et al also demonstrated that inhibiting SIRT1 may conserve cellular $\mathrm{NAD}^{+}$levels and protect neurons against ischemic damage. Additionally, SIRT1 may play a dual role, protective as well as destructive, in Huntington's disease $(52,53)$. However, the results of the present study suggest that HPC exert neuroprotective effects by activating the SIRT1/FoxO1 pathway.

It has been demonstrated that upregulating the level of autophagy in neurons can reduce cell death and improve recovery. HPC has emerged as a viable new therapy for GCI, offering a non-invasive approach to reducing neurological injury. However, there are several pathways that mediate autophagy, and the SIRT1/FoxO1 pathway is only one of them, so it cannot be definitively concluded that the neuroprotective effects were entirely due to HPC. Additional studies are needed to confirm the current results and elucidate the mechanisms underlying the neuroprotective properties of HPC.

In conclusion, taken together, the data of the present study indicated that HPC treatment exerts beneficial effects on neuronal survival and preservation of cognitive function following GCI, which may be mediated via activation of the SIRT1/FoxO1 pathway that increases neuronal autophagy. Based on these beneficial effects, and the fact that HPC is a non-invasive and cost-effective therapy, HPC may hold promise as a potential treatment in humans for ameliorating the poor cognitive outcomes following GCI.

\section{Acknowledgements}

Not applicable.

\section{Funding}

The present study was supported by the Hebei Medical Science Research Project, China (grant no. 20190106); the Tangshan City Science and Technology Project, China (grant no. 18130232a); and the North China University of Science and Technology Research and Research Project (grant no. Z201736).

\section{Availability of materials and data}

The datasets used and/or analyzed during the current study are available from the corresponding author on reasonable request.

\section{Authors' contributions}

JL and YZ designed the study; JL, JW, XW and JW performed the experiments; JX analyzed the data; SL complementary experiments; JL and ZG wrote the manuscript. All the authors have read and approved the final manuscript.

\section{Ethics approval and consent to participate}

All procedures used in the present study were approved by the Institutional Animal Care and Use Committee of North China University of Science and Technology (no. 2015-99) and were conducted in accordance with the guidelines of the National Institutes of Health of the United State of America for animal research.

\section{Patient consent for publication}

Not applicable.

\section{Competing interests}

The authors declare that they have no competing interests.

\section{References}

1. Jalife J: The tornadoes of sudden cardiac arrest. Nature 555: 597-598, 2018.

2. Yeung J and Moulaert V: Focus on the brain of the cardiac arrest survivor. Resuscitation 88: A5-A6, 2015.

3. Weihs W, Warenits AM, Ettl F, Magnet IA, Teubenbacher U, Hilpold A, Schober A, Testori C, Tiboldi A, Mag KT, et al: Reduced long-term memory in a rat model of 8 minutes ventricular fibrillation cardiac arrest: A pilot trial. BMC Vet Res 12: 103, 2016.

4. Woods D and Chantavarin S: Serial neuropsychological assessment of an adolescent girl after suffering a sudden out-of-hospital-cardiac-arrest following recreational inhalant use. Appl Neuropsychol Child 6: 378-387, 2017.

5. Wolman RL, Nussmeier NA, Aggarwal A, Kanchuger MS, Roach GW, Newman MF, Mangano CM, Marschall KE, Ley C, Boisvert DM, et al: Cerebral injury after cardiac surgery: Identification of a group at extraordinary risk. Multicenter Study of Perioperative Ischemia Research Group (McSPI) and the ischemia research education foundation (IREF) investigators. Stroke 30: 514-522, 1999.

6. Tsunekawa T, Sawada M, Kato T, Motoji Y, Kinoshita T, Hirakawa A, Okawa Y and Tomita S: The prevalence and distribution of occlusive lesions of the cerebral arteries in patients undergoing coronary artery bypass graft surgery. Semin Thorac Cardiovasc Surg 30: 413-420, 2018.

7. Ji NN, Wu L, Shao BM, Meng QX, Xu JN, Zhu HW and Zhang YM: CTL-Derived Granzyme B participates in hippocampal neuronal apoptosis induced by cardiac arrest and resuscitation in rats. Front Neurol 10: 1306, 2019.

8. Lee D, Pearson T, Proctor JL, Rosenthal RE and Fiskum G: Oximetry-Guided normoxic resuscitation following canine cardiac arrest reduces cerebellar Purkinje neuronal damage. Resuscitation 140: 23-28, 2019.

9. Harukuni I and Bhardwaj A: Mechanisms of brain injury after global cerebral ischemia. Neurol Clin 24: 1-21, 2006.

10. Kim YM, Yim HW, Jeong SH, Klem ML and Callaway CW: Does therapeutic hypothermia benefit adult cardiac arrest patients presenting with non-shockable initial rhythms? A systematic review and meta-analysis of randomized and non-randomized studies. Resuscitation 83: 188-196, 2012.

11. Kim KA, Kim D, Kim JH, Shin YJ, Kim ES, Akram M, Kim EH, Majid A, Baek SH and Bae ON: Autophagy-mediated occludin degradation contributes to blood-brain barrier disruption during ischemia in bEnd.3 brain endothelial cells and rat ischemic stroke models. Fluids Barriers CNS 17: 21, 2020.

12. Chen R, Zhang YY, Lan JN, Liu HM, Li W, Wu Y, Leng Y, Tang LH, Hou JB, Sun Q, et al: Ischemic postconditioning alleviates intestinal ischemia-reperfusion injury by enhancing autophagy and suppressing oxidative stress through the Akt/GSK-3 $\beta /$ Nrf2 pathway in mice. Oxid Med Cell Longev 2020: 6954764,2020 . 
13. Liu Z, Zhang J,Zhang F and Chang Y: Propofol post-conditioning lessens renal ischemia/reperfusion-induced acute lung injury associated with autophagy and apoptosis through MAPK signals in rats. Gene 741: 144562, 2020.

14. Wu B, Luo H, Zhou X, Cheng CY, Lin L, Liu BL, Liu K, Li P and Yang H: Succinate-induced neuronal mitochondrial fission and hexokinase II malfunction in ischemic stroke: Therapeutical effects of kaempferol. Biochim Biophys Acta Mol Basis Dis 1863: 2307-2318, 2017.

15. Wu X, He L, Cai Y, Zhang G, He Y, Zhang Z, He X, He Y, Zhang $G$ and Luo J: Induction of autophagy contributes to the myocardial protection of valsartan against ischemia reperfusion injury. Mol Med Rep 8: 1824-1830, 2013.

16. Lv B, Li F, Han J, Xu L, Sun C, Hua T, Zhang Z, Feng Z and Jiang X: Hif- $1 \alpha$ overexpression improves transplanted bone mesenchymal stem cells survival in rat MCAO stroke model. Front Mol Neurosci 10: 80, 2017.

17. Wang P, Xu TY, Wei K, Guan YF, Wang X, Xu H, Su DF, Pei G and Miao CY: ARRB1/ $\beta$-arrestin-1 mediates neuroprotection through coordination of BECN1-dependent autophagy in cerebral ischemia. Autophagy 10: 1535-1548, 2014.

18. Deng Z, Li Y, Liu H, Xiao S, Li L, Tian J, Cheng C, Zhang G and Zhang F: The role of sirtuin 1 and its activator, resveratrol in osteoarthritis. Biosci Rep 39: BSR20190189, 2019.

19. Cao W, Dou Y and Li A: Resveratrol boosts cognitive function by targeting SIRT1. Neurochem Res 43: 1705-1713, 2018.

20. Gonfloni S, Iannizzotto V, Maiani E, Bellusci G, Ciccone S and Diederich M: P53 and Sirt1: Routes of metabolism and genome stability. Biochem Pharmacol 92: 149-156, 2014.

21. Hwang JW, Yao H, Caito S, Sundar IK and Rahman I: Redox regulation of SIRT1 in inflammation and cellular senescence. Free Radic Biol Med 61: 95-110, 2013.

22. Khan M, Ullah R, Rehman SU, Shah SA, Saeed K, Muhammad T, Park HY, Jo MH, Choe K, Rutten BPF and Kim MO: 17 $\beta$-estradiol modulates SIRT1 and halts oxidative stress-mediated cognitive impairment in a male aging mouse model. Cells 8: 928, 2019.

23. Tang X, Zhao Y, Zhou Z, Yan J, Zhou B, Chi X, Luo A and Li S: Resveratrol mitigates sevoflurane-induced neurotoxicity by the SIRT1-dependent regulation of BDNF expression in developing mice. Oxid Med Cell Longev 2020: 9018624, 2020.

24. Nikseresht S, Khodagholi F and Ahmadiani A: Protective effects of ex-527 on cerebral ischemia-reperfusion injury through necroptosis signaling pathway attenuation. J Cell Physiol 234: 1816-1826, 2019.

25. Huang J, Tian R, Yang Y, Jiang R, Dai J, Tang L and Zhang L: The SIRT1 inhibitor EX-527 suppresses mTOR activation and alleviates acute lung injury in mice with endotoxiemia. Innate Immun 23: 678-686, 2017.

26. Calnan DR and Brunet A: The FoxO code. Oncogene 27: 2276-2288, 2008.

27. Yan X, Yu A, Zheng H, Wang S, He Y and Wang L: Calycosin-7-O- $\beta$-D-glucoside attenuates OGD/R-induced damage by preventing oxidative stress and neuronal apoptosis via the SIRT1/FOXO1/PGC-1 $\alpha$ pathway in HT22 cells. Neural Plast 2019: 8798069, 2019.

28. Susanti VY, Sasaki T, Yokota-Hashimoto H, Matsui S, Lee YS, Kikuchi O, Shimpuku M, Kim HJ, Kobayashi M and Kitamura T: Sirtl rescues the obesity induced by insulin-resistant constitutively-nuclear FoxO1 in POMC neurons of male mice. Obesity (Silver Spring) 22: 2115-2119, 2014.

29. Fan L, Chen D, Wang J, Wu Y, Li D and Yu X: Sevoflurane ameliorates myocardial cell injury by inducing autophagy via the deacetylation of LC3 by SIRT1. Anal Cell Pathol (Amst) 2017: 6281285, 2017

30. Zhang Z, Li D, Xu L and Li HP: Sirt1 improves functional recovery by regulating autophagy of astrocyte and neuron after brain injury. Brain Res Bull 150: 42-49, 2019.

31. Wang Y, Hao Y, Zhang H, Xu L, Ding N, Wang R, Zhu G, Ma S, Yang A, Yang Y, et al: DNA hypomethylation of miR-30a mediated the protection of hypoxia postconditioning against aged cardiomyocytes hypoxia/reoxygenation injury through inhibiting autophagy. CIRC J 84: 616-625, 2020.

32. Albrecht M,Zitta K, GroenendaalF, van BelFand Peeters-Scholte C: Neuroprotective strategies following perinatal hypoxia-ischemia: Taking aim at NOS. Free Radic Biol Med 142: 123-131, 2019.

33. Nguyen HL, Ruhoff AM, Fath T and Jones NM: Hypoxic postconditioning enhances functional recovery following endothelin-1 induced middle cerebral artery occlusion in conscious rats. Exp Neurol 306: 177-189, 2018
34. Zhan L, Li D, Liang D, Wu B, Zhu P, Wang Y, Sun W and Xu E: Activation of Akt/FoxO and inactivation of MEK/ERK pathways contribute to induction of neuroprotection against transient global cerebral ischemia by delayed hypoxic postconditioning in adult rats. Neuropharmacology 63: 873-882, 2012.

35. Zhu P, Zhan L, Zhu T, Liang D, Hu J, Sun W, Hou Q, Zhou H, Wu B, Wang Y and Xu E: The roles of p38 MAPK/MSK1 signaling pathway in the neuroprotection of hypoxic postconditioning against transient global cerebral ischemia in adult rats. Mol Neurobiol 49: 1338-1349, 2014.

36. Tu J, Zhang X, Zhu Y, Dai Y, Li N, Yang F, Zhang Q, Brann DW and Wang R: Cell-permeable peptide targeting the Nrf2-Keap1 Interaction: A potential novel therapy for global cerebral ischemia. J Neurosci 35: 14727-14739, 2015.

37. Rybnikova E, Vorobyev M, Pivina S and Samoilov M: Postconditioning by mild hypoxic exposures reduces rat brain injury caused by severe hypoxia. Neurosci Lett 513: 100-105, 2012.

38. Zhang HL, Xu M, Wei C, Qin AP, Liu CF, Hong LZ, Zhao XY, Liu J and Qin ZH: Neuroprotective effects of pioglitazone in a rat model of permanent focal cerebral ischemia are associated with peroxisome proliferator-activated receptor gamma-mediated suppression of nuclear factor- $\kappa \mathrm{B}$ signaling pathway. Neuroscience 176: 381-395, 2011.

39. Kirino T and Sano K: Selective vulnerability in the gerbil hippocampus following transient ischemia. ACTA Neuropathol 62: 201-208, 1984.

40. Chen J, Zhu RL, Nakayama M, Kawaguchi K, Jin K, Stetler RA, Simon RP and Graham SH: Expression of the apoptosis-effector gene, Bax, is upregulated in vulnerable hippocampal CA1 neurons following global ischemia. J Neurochem 67: 64-71, 1996.

41. Zhu T, Zhan L, Liang D, Hu J, Lu Z, Zhu X, Sun W, Liu L and $\mathrm{Xu}$ E: Hypoxia-inducible factor $1 \alpha$ mediates neuroprotection of hypoxic postconditioning against global cerebral ischemia. J Neuropathol Exp Neurol 73: 975-986, 2014

42. Solevag AL, Schmolzer GM and Cheung PY: Novel interventions to reduce oxidative-stress related brain injury in neonatal asphyxia. Free Radic Biol Med 142: 113-122, 2019.

43. Wolf MS, Bayir H, Kochanek PM and Clark R: The role of autophagy in acute brain injury: A state of flux? Neurobiol Dis 122: 9-15, 2019.

44. Mizushima N: Autophagy: Process and function. Genes Dev 21: 2861-2873, 2007.

45. Baehrecke EH: Autophagy: Dual roles in life and death? Nat Rev Mol Cell Biol 6: 505-510, 2005.

46. Ryan F, Khodagholi F, Dargahi L, Minai-Tehrani D and Ahmadiani A: Temporal pattern and crosstalk of necroptosis markers with autophagy and apoptosis associated proteins in ischemic hippocampus. Neurotox Res 34: 79-92, 2018.

47. Marzetti E, Calvani R, Cesari M, Buford TW, Lorenzi M, Behnke BJ and Leeuwenburgh C: Mitochondrial dysfunction and sarcopenia of aging: From signaling pathways to clinical trials. Int J Biochem Cell Biol 45: 2288-2301, 2013.

48. Martinez MA, Rodriguez JL, Lopez-Torres B, Martínez M, Martínez-Larrañaga MR, Maximiliano JE, Anadón A and Ares I: Use of human neuroblastoma SH-SY5Y cells to evaluate glyphosate-induced effects on oxidative stress, neuronal development and cell death signaling pathways. Environ Int 135: 105414, 2020.

49. Lim CJ, Lee YM, Kang SG, Lim HW, Shin KO, Jeong SK, Huh YH, Choi S, Kor M, Seo HS, et al: Aquatide activation of SIRT1 reduces cellular senescence through a SIRT1FOXO1-autophagy axis. Biomol Ther (Seoul) 25: 511-518,2017.

50. Jiang X, Niu XL, Guo Q, et al: FoxO1-mediated autophagy plays an important role in the neuroprotective effects of hydrogen in a rat model of vascular dementia. Behav Brain Res 356: 98-106, 2019.

51. Wu B, Feng JY, Yu LM, Wang YC, Chen YQ, Wei Y, Han JS, Feng X, Zhang Y, Di SY, et al: Icariin protects cardiomyocytes against ischaemia/reperfusion injury by attenuating sirtuin 1-dependent mitochondrial oxidative damage. Br J Pharmacol 175: 4137-4153, 2018

52. Jiang M, Wang J, Fu J, Du L, Jeong H, West T, Xiang L, Peng Q, Hou Z, Cai H, et al: Neuroprotective role of Sirt1 in mammalian models of Huntington's disease through activation of multiple Sirt1 targets. Nat Med 18: 153-158, 2011.

53. Süssmuth SD, Haider S, Landwehrmeyer GB, Farmer R, Frost C, Tripepi G, Andersen CA, Di Bacco M, Lamanna C, Diodato E, et al: An exploratory double-blind, randomized clinical trial with selisistat, a SirT1 inhibitor, in patients with Huntington's disease. Brit J Clin Pharmaco 79: 465-476, 2015.

This work is licensed under a Creative Commons Attribution-NonCommercial-NoDerivatives 4.0 International (CC BY-NC-ND 4.0) License. 